\title{
Infrasonic Observations of Meteoroids: Preliminary Results from a Coordinated Optical-radar-infrasound Observing Campaign
}

\author{
Wayne N. Edwards · Peter G. Brown · Robert J. Weryk · Douglas O. ReVelle
}

Received: 27 June 2007/ Accepted: 12 September 2007/Published online: 12 October 2007

(C) Springer Science+Business Media B.V. 2007

\begin{abstract}
Recent observations using the newly installed Elginfield infrasound array in coordination with the Southern Ontario all-sky meteor camera network and Canadian Meteor Orbit Radar (CMOR) has shown that the number of meteors producing infrasound at the Earth's surface is more frequent than previously thought. These data show the flux of meteoroids capable of producing infrasound at the ground is at least $1 / \mathrm{month}$ and is limited to meteors with peak visual brightness above -2 . Comparisons to current meteor infrasound theory show excellent agreement with amplitude and period predictions for weakly non-linear shock waves using a realistic vertically inhomogeneous atmosphere. Similar predictions show isothermal assumptions underestimate the amplitude by orders of magnitude.
\end{abstract}

Keywords Atmosphere $\cdot$ Infrasound $\cdot$ Meteor $\cdot$ Meteoroid $\cdot$ Shock waves

\section{Introduction}

Observations of infrasonic sound from large $>1 \mathrm{~m}$ diameter meteoroids are well documented and are becoming more common with the inception of the International Monitoring System's (IMS) global network of infrasound stations. This coincides with a general revival of infrasound as a monitoring tool (e.g. ReVelle 1997; Brown et al. 2002;

\footnotetext{
W. N. Edwards $(\bowtie)$

Department of Earth Sciences, University of Western Ontario, 1151 Richmond Street, London, ON, Canada N6A 5B7

e-mail: wedwards@uwo.ca

P. G. Brown · R. J. Weryk

Department of Physics and Astronomy, University of Western Ontario, 1151 Richmond Street, London, ON, Canada N6A 3K7

D. O. ReVelle

Atmospheric, Climate and Environmental Dynamics, Meteorological Modeling Team, Los Alamos National Laboratory, MS D401, P.O. Box 1663, Los Alamos, NM 87545, USA
} 
Klekociuk et al. 2005; Edwards et al. 2006). Yet similar infrasonic observations of smaller ( $<10 \mathrm{~cm}$ diameter) meteoroids remain rare: only a handful of these are fully documented (Kraemer 1977; Brown et al. 2007). Other observations of infrasound from small meteoroids tend to be coincident signals that are detected after a bright meteor event but lack trajectory information - this limits confidence in these associations (McIntosh et al. 1976; ReVelle and Whitaker 1999; Le Pichon 2002). With the sparseness of this dataset the result is that fundamental meteor infrasound theory (ReVelle 1974, 1976) and its predictions have remained generally untested and unconstrained by observation for more than 30 years.

To address this need, an ongoing coordinated campaign is underway using the Southern Ontario Meteor Network's (SOMN) all-sky camera system, the Canadian Meteor Orbit Radar (CMOR) and the newly installed Elginfield infrasonic array (ELFO) to monitor meteor generated infrasound from common regional meteor events. Such events refer to modestly bright fireballs ( -2 and brighter) and have the advantage that they are at close range to the observing station such that the acoustic signal is not significantly attenuated or dispersed. Typical ground-projected distances are within $\sim 200 \mathrm{~km}$ (depending on meteor altitude) or before the acoustic/infrasonic sound refracts back up into the atmosphere due to the tropospheric temperature/sound speed gradient. The goal of this long-term campaign is to determine or constrain: (1) the flux of infrasound producing meteoroids at the Earth's surface, (2) the altitudes at which these infrasonic waves are being generated, (3) the fundamental physics of shock production during hypersonic flight of meteoroids and (4) the relationship between a meteoroid's kinetic energy and surface observations of period and amplitude. The following sections will describe the preliminary results of the first $1 \frac{1}{2}$ years of this campaign.

\section{Equipment and Methodology}

The equipment used in this campaign are all component parts of the SOMN. This includes six all-sky video meteor cameras (Weryk et al. 2007), CMOR (Jones et al. 2005) and the four element infrasound station ELFO located in Elginfield, Ontario $\left(43.1907^{\circ} \mathrm{N}\right.$, $81.3152^{\circ} \mathrm{W}, 322 \mathrm{~m}$, Fig. 1). These first two systems provide triggers for potential infrasound producing meteors and direct when to search for signals in the continuous pressure data logged by ELFO. In the case of the SOMN all-sky cameras, these triggers are bright visual meteors $\left(\mathrm{M}_{\mathrm{P}}<-2\right)$, while for CMOR, they are obvious cases of meteor head-echo detection (i.e. radar reflections from the plasma surrounding the meteoroid which are automatically detected at a rate of $\sim 3$ /week).

In the case of potential meteor detection the analysis methodology to confirm the observation and detection is as follows:

(1) All-sky camera (multi-station): Event time, trajectory, radiant, light curve, velocity and mass are determined using standard reduction methods (Ceplecha and McCrosky 1976; Ceplecha 1987; Ceplecha et al. 1998; Borovička 1990).

(2) All-sky camera (single-station): Event time and direction are determined.

(3) Radar detection: Event time, range rate, duration and signal interferometry are recorded and used to determine $3 \mathrm{D}$ meteor trajectory and velocity.

(4) Comparison: Observed infrasonic back azimuth and propagation time are compared to the range and direction to the observed meteor trajectory. If these are found to be consistent with the meteor source, the event is logged as a probable detection. 


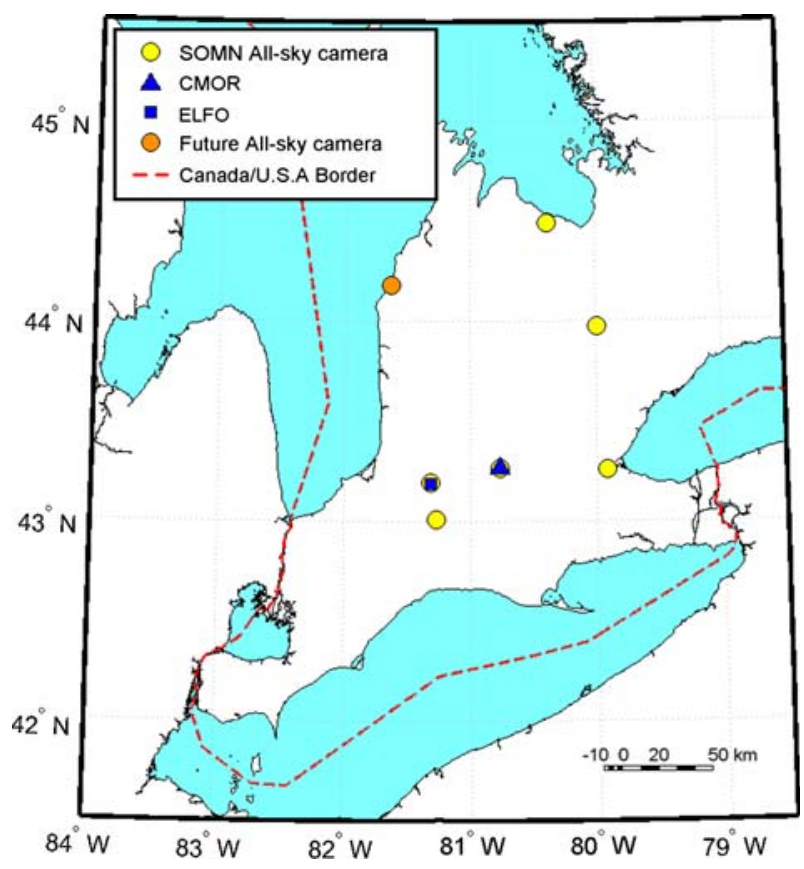

Fig. 1 Map of the Southern Ontario Meteor Network of all-sky cameras and relative locations of CMOR and the Elginfield observatory infrasound array, ELFO

(5) Reconstruction: Atmospheric data collected includes: (a) UKMO stratospheric assimilated data (Swinbank and O'Neill 1994) profile for the day of observation, (b) mesospheric winds measured from $82-98 \mathrm{~km}$ by CMOR (Hocking 1997), (c) MSISE-00 (Hedin 1991) and HWM93 (Hedin et al. 1996) atmospheric temperature, pressure and horizontal wind models. These measurements/models are fused together and used to reconstruct the best fit atmospheric propagation conditions at the time of the infrasonic detection.

(6) Ray tracing: Using the observed locations on the trajectory, rays are propagated towards ELFO, through the reconstructed atmosphere and the resulting travel times, azimuths and incidence angles are compared to those observed (Edwards and Hildebrand 2004). If a solution exists which reproduces the observations well (travel time delay, arrival azimuth, arrival angle), the observation is then confirmed and the source altitude delimited.

\section{Observations}

Between January 23, 2006 and June 6, 2007 (nearly 17 months of operation at ELFO) the total number of probable infrasound detection from regional meteors is 18 . Of these 18 events, 13 have been coincident with a multi-station meteor detection affording complete trajectory and mass/energy estimation. Details of the detections are given in Table 1. Even with slightly more than a dozen confirmed multi-camera detections one significant conclusion can be drawn; infrasound from moderately sized ( $<10 \mathrm{~cm}$ diameter) is significantly 


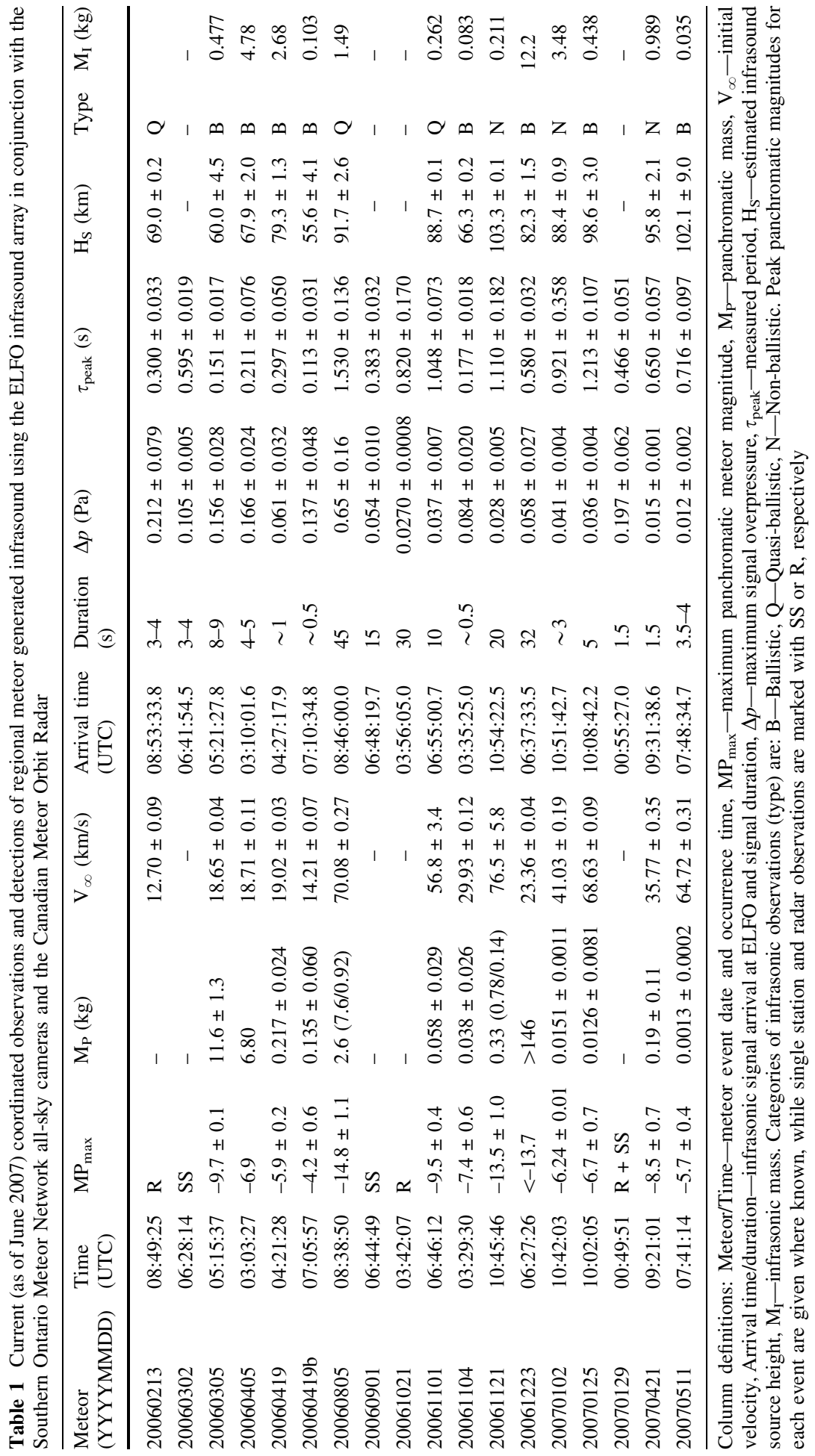


more common than has been previously observed. Previous attempts at monitoring regional meteor infrasound at the Springhill Meteor Observatory (McIntosh et al. 1976), and by the Prairie Network (Kraemer 1977) resulted in only one confirmed sporadic meteor and one probable Geminid meteor infrasonic signal after $\sim 5$ years of observation. Our current observations show that the flux is at least two orders of magnitude higher than these early observations suggest. We suspect that significant advances in computer technology and digital signal processing since the late 1970s and early 1980s, has likely made identification of these sometimes very weak signals (Table 1) far easier.

The bulk of the observed detections in Table 1 tend to be from sporadic meteors. Some shower meteors, however, have been identified and include a suspected Orionid (20061021) and a confirmed Leonid (20061121) and Quadrantid (20070102). Additionally upon inspection of ray tracing geometry (i.e. deviation of the ray vector from the meteor trajectory), the observations may be placed into one of three categories:

(1) Ballistic-rays which propagate approximately $90^{\circ} \pm 20^{\circ}$ (Brown et al. 2007) from the meteor trajectory, consistent with cylindrical blast wave theory (ReVelle 1976).

(2) Quasi-ballistic — rays which border, but do not fall within, the ballistic regime $\left(110^{\circ}-\right.$ $125^{\circ}$ deviation). Although often appearing to have ballistic shock features (i.e. N-type waves), these deviations are sufficiently large that they cannot be categorized as ballistic waves within uncertainties in the trajectory or model atmosphere.

(3) Non-ballistic - rays which appear to emit from an omni-directional or point-like source (e.g. due to fragmentation or the blunt end of a meteoroid's hypersonic shock).

Having identified potential source mechanisms for the observed meteor infrasound, we attempt to reproduce the ballistic and quasi-ballistic observations using current theory constrained by the known trajectory \& source regions (ReVelle 1974, 1976).

\section{Comparison with Theory}

The theoretical developments of meteor generated infrasound were developed initially by ReVelle (1974) based upon research in weak shock propagation and cylindrical blast waves and applying these to high altitude, hypersonic meteor sources. While initial treatments (ReVelle 1976) were based upon simplifying isothermal atmosphere assumptions, a method was provided as to how the theory could be extended to more realistic vertically inhomogeneous atmospheres (ReVelle 1974). Using a top down methodology, we use the ray geometry and the observed meteor trajectory, photometric mass and velocity to calculate the predicted theoretical signal overpressure (amplitude), $\Delta p$, and period using both an isothermal and vertically inhomogeneous atmosphere.

Theoretical results for the nominal geometries for ballistic and quasi-ballistic observations are shown in Fig. 2. Theoretical uncertainties shown are the variation in predicted $\Delta p$ and period accounting for uncertainties in geometry, mass and velocity. While predicted periods show similar scatter about the observed periods on the order of factors of 2 or 3 for either model atmosphere (Fig. 2b/d), due to general insensitivity of the theoretical period on model conditions (see ReVelle 1976 Eqs. 15 and 34), predicted weak shock $\Delta p$ show significant differences (Fig. 2a/c). Using an isothermal atmosphere, $\Delta p$ is consistently underestimated by at least two orders of magnitude if treated as a propagating weak shock wave. A vertically inhomogeneous, however, (thermally stratified, range independent) atmosphere produces similar agreement in $\Delta p$ as seen for period. Treating the propagating wave as a weak shock that transitions to a linear wave (not shown) produces 

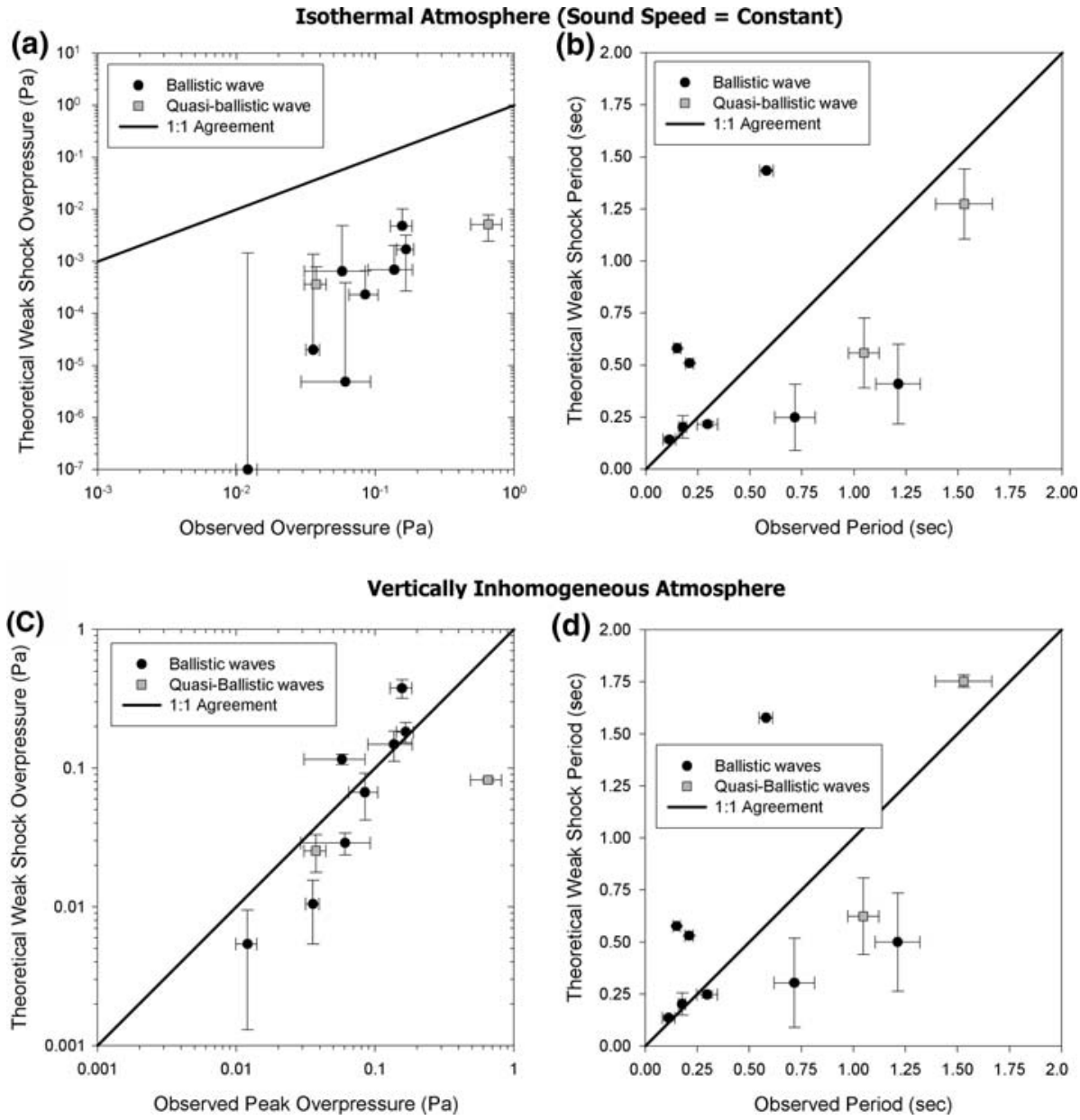

Fig. 2 Comparison of cylindrical weak shock theory of meteor generated infrasound (ReVelle 1974, 1976) with the observations of ballistic and quasi-ballistic infrasound at ELFO for those meteors with known trajectories. (a/b) For an isothermal atmosphere and (c/d) vertically inhomogeneous (layered) atmosphere

$\Delta p$ 's for isothermal and inhomogeneous models that consistently underestimate and overestimate, respectively, the observed $\Delta p$ by at least an order of magnitude, due to the slower decay of linear waves over weak shocks $\left(\mathrm{x}^{-1 / 2}\right.$ vs. $\mathrm{x}^{-3 / 4}$ respectively where $\mathrm{x}$ is scaled distance from the source, $R / R_{\mathrm{o}}$ ).

We conclude, therefore, that observations of regional meteor infrasound so far recorded by ELFO are consistent with theory assuming predominantly weakly non-linear shock waves propagate to the ground. Knowing this we attempt a bottom-up treatment, determining the mass of each source meteoroid (independent from photometric methods) by least squares fitting the sum of the residuals between theoretical $\Delta p$ and period and those observed, using vertically inhomogeneous cylindrical blast wave theory with the meteoroid mass as the sole variable (the remaining parameters being constrained by observation). The resulting infrasonic mass for ballistic and quasi-ballistic observations (Table 1) is found to relate to the photometric mass roughly as: 
Fig. 3 Comparison of infrasonic mass to panchromatic mass. Infrasonic mass determined by fitting theoretical signal amplitude and period to observed signal. Panchromatic mass determined through standard light curve analysis (Ceplecha et al. 1998)

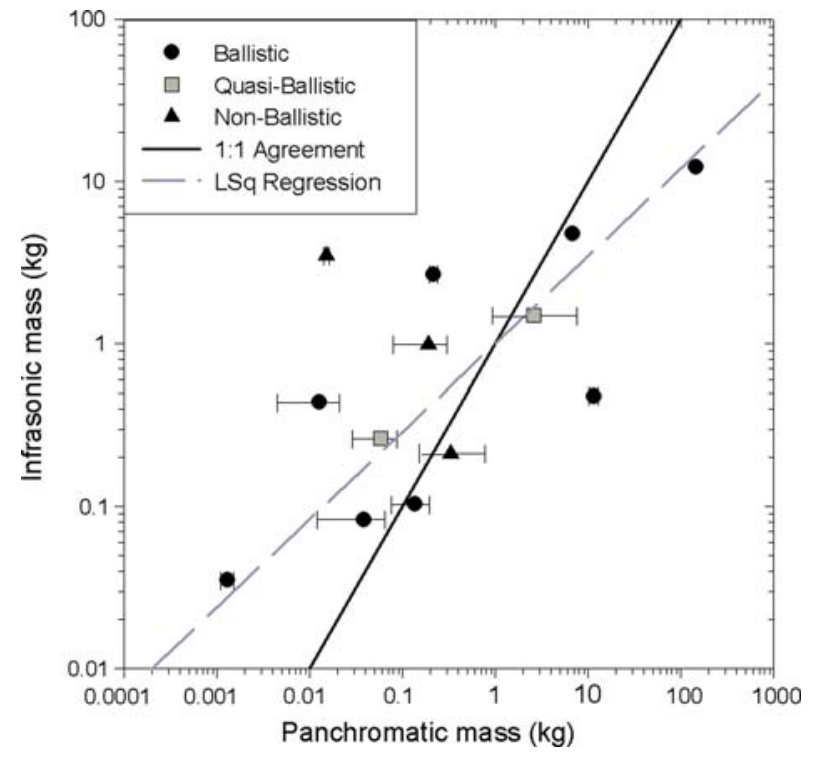

$M_{I}=1.00 \pm 0.60 M_{P}^{0.54 \pm 0.12}$

where $M_{I}$ is the infrasonic mass and $M_{P}$ is the photometric mass in kilograms (Fig. 3).

\section{Discussion and Conclusions}

Recent observations of regional meteor infrasound using the newly installed ELFO infrasound array in tandem with the SOMN all-sky cameras and CMOR have discovered that the flux of meteor generated infrasound from small meteoroids $<10 \mathrm{~cm}$ in diameter is far higher than previously observed. Although final calibration of the flux is in progress (awaiting corrections for observing biases and sky coverage), the flux appears to be at least 1 event/ month for meteor panchromatic magnitudes -2 and brighter. Interestingly, ReVelle (1974) predicted $\sim 20$ events in a winter season might be detectable. The current results are a lower limit as no daytime events or meteors occurring during bad weather are detected. This suggests that ReVelle's (1974) estimate may be quite close to the actual effective detection limit. Work continues on correlating radar observed meteor head-echoes with the infrasound, preliminary results suggesting a handful of positive correlations/detections.

Recent observations using the European Fireball Network (Oberst et al. 1998) and the IMS station I26DE (Brown et al. 2007) showed that high altitude infrasound from shower meteors was possible. This more complete and general survey suggests that not only is infrasound production possible at these altitudes, it appears that it is quite common for meteors $<-4$ magnitude with at least 8 of 18 signals originating at altitudes $>80 \mathrm{~km}$. Similar to the results of Brown et al. (2007), ballistic shocks make up the majority of observations with non-ballistic observations being far more rare and often associated with meteors undergoing gross fragmentation.

Using the current observations of ballistic and quasi-ballistic meteor generated infrasound we are at last able to begin testing and constraining the theoretical predictions developed by ReVelle (1974) more than 30 years ago. We find that most of our 
observations fit with ELFO recording the arrival of weakly non-linear shock waves. Yet only when using a realistic vertically inhomogeneous atmosphere can the theory reasonably fit the overpressure observations. Isothermal atmosphere assumptions underestimate this amplitude by at least two orders of magnitude. Such a significant finding implies that the kinetic energy (E)-overpressure relationship (Ceplecha et al. 1998),

$$
E=11.5 \pi \rho_{m} R^{\prime 3}\left(\Delta p / \sqrt{p_{Z} p_{g}}\right)^{4} \frac{C_{S}^{3}}{V}
$$

first introduced by (Brown et al. 1996) and based upon these isothermal assumptions, will significantly over estimate meteoroid kinetic energy by orders of magnitude when observed $\Delta p$ are input. Note that in (2) and later (3), $\rho_{m}$, is the meteoroid density; $V$, meteoroid velocity; $R^{\prime}$ is the range from observation point to the meteor and $p_{Z}$ and $p_{g}$ are the ambient pressures at the source height and ground, respectively. Such over-the-top and/or highly variable estimates of kinetic energy have been commonly reported when applying this formula to real data (e.g. Brown et al. 1996; ReVelle and Whitaker 1999; Brown et al. 2007). In contrast, the equivalent kinetic energy-period $(\tau)$ relationship (Ceplecha et al. 1998),

$$
E=\left(\frac{\pi}{12}\right)\left(\frac{\tau}{1.579}\right)^{4}\left(\frac{\rho_{m} C_{S}^{7}}{V R^{\prime}}\right)
$$

also based upon isothermal assumptions, should produce more robust energy estimates than overpressure despite the severe dependence on the sound speed, $C_{S}$. This is due in part to the relative insensitivity of the period (in the current theory) to the atmospheric model chosen (ReVelle 1974, 1976) and because the speed of sound varies relatively little $(305 \mathrm{~m} / \mathrm{s} \pm 15 \%)$ between $\sim 120$ and $50 \mathrm{~km}$ altitude, where most of the meteor infrasound observed so far is generated (Table 1).

As the current multi-instrumental campaign to observe infrasound from these smaller, more common, regional meteors continues, it will finally be possible to explore the infrasound and hyper-velocity meteoroid relationship both quantitatively and statistically, placing limits on current theory and potentially revising our current understanding of the shock mechanism at the source. Additionally, further work on ballistic observations should also provide another method to constrain other poorly known quantities in hyper-velocity meteoroid-atmosphere interactions such as luminous efficiency and bulk density through use of infrasonic energy estimates and infrasonic mass in tandem with electro-optical observations. This should be possible for ballistic observations since, due to cylindrical propagation, ballistic waves originate along a finite section of the meteor trail and thus be related to the physical properties of the meteoroid (mass/size) along that section. Infrasound in meteor science is now steadily progressing from its early theoretical confines to both observational and practical applications.

\section{References}

J. Borovička, The comparison of two methods of determining meteor trajectories from photographs. Bull. Astron. Inst. Czech. 41, 391-396 (1990)

P. Brown, A.R. Hildebrand, D.W.E. Green, D. Pagé, C. Jacobs, D. ReVelle, E. Tagliaferri, J. Wacker, B. Wetmiller, The fall of the St-Robert meteorite. Meteorit. Planet. Sci. 31, 502-517 (1996)

P.G. Brown, R.W. Whitaker, D.O. ReVelle, E. Tagliaferri, Multi-station infrasonic observations of two bolides: signal interpretation and implications for monitoring of atmospheric explosions. Geophys. Res. Lett. 29 (2002). doi: 10.1029/2001GL013778 
P.G. Brown, W.N. Edwards, D.O. ReVelle, P. Spurny, Acoustic analysis of shock production by very highaltitude meteors-I: infrasonic observations, dynamics and luminosity. J. Atmos. Solar-Terres. Phys. 69, 600-620 (2007)

Z. Ceplecha, Geometric, dynamic, orbital and photometric data on meteoroids from photographic fireball networks. Bull. Astron. Inst. Czech. 38, 222-234 (1987)

Z. Ceplecha, R.E. McCrosky, Fireball end heights: a diagnostic for the structure of meteoric material, J. Geophys. Res. 81, 6257-6275 (1976)

Z. Ceplecha, J. Borovička, W.G. Elford, D.O. ReVelle, R.L. Hawkes, V. Porubčan, M. Šimek, Meteor phenomena and bodies. Space Sci. Rev. 84, 327-471 (1998)

W.N. Edwards, A.R. Hildebrand, SUPRACENTER: Locating fireball terminal bursts in the atmosphere using seismic arrivals, Meteorit. Planet. Sci. 39, 1449-1460 (2004)

W.N. Edwards, P.G. Brown, D.O. ReVelle, Estimates of meteoroid kinetic energies from observations of infrasonic airwaves. J. Atmos. Solar-Terres. Phys. 68, 1136-1160 (2006)

A.E. Hedin, Extension of the MSIS thermospheric model into the middle and lower atmosphere. J. Geophys. Res. 96, 1159-1172 (1991)

A.E. Hedin, E.L. Fleming, A.H. Manson, F.J. Schmidlin, S.K. Avery, R.R. Clark, S.J. Franke, G.J. Fraser, T. Tsuda, F. Vial, R.A. Vincent, Empirical wind model for the upper, middle and lower atmosphere. J. Atmos. Terres. Phys. 58, 1421-1447 (1996)

W.K. Hocking, Strengths and limitations of MST radar measurements of middle-atmosphere winds. Ann. Geophys. 15, 1111-1122 (1997)

J. Jones, P. Brown, K.J. Ellis, A.R. Webster, M. Campbell-Brown, Z. Krzemenski, R.J. Weryk, The Canadian Meteor Orbit Radar: system overview and preliminary results. Planet. Space Sci. 53, 413-421 (2005)

A.R. Klekociuk, P.G. Brown, D.W. Pack, D.O. ReVelle, W.N. Edwards, R.E. Spalding, E. Tagliaferri, Y.B. Bernard, J. Zagari, Lidar, satellite and acoustic measurements of an asteroidal airburst in Earth's atmosphere. Nature 436, 1132-1135 (2005)

D.R. Kraemer, Infrasound from accurately measured meteor trails, Ph.D. Dissertation, Ann Arbor, University of Michigan, MI (1977)

A. Le Pichon, J.M. Guerin, E. Blanc, D. Raymond, Trail in the atmosphere of the 29 December 2000 meteor as recorded in Tahiti: characteristics and Trajectory reconstitution, J. Geophys. Res. 107 (2002). doi: $10.1029 / 2001$ JD001283

B.A. McIntosh, M.D. Watson, D.O. ReVelle, Infrasound from a radar-observed meteor. Can. J. Phys. 54, 655-662 (1976)

J. Oberst, S. Molau, D. Heinlein, C. Gritzner, M. Schindler, P. Spurny, Z. Ceplecha, J. Rendtel, H. Betlem, The "European Fireball Network": current status and future prospects. Meteorit. Planet. Sci. 33, 49-56 (1998)

D.O. ReVelle, Acoustics of meteors-effects of the atmospheric temperature and wind structure on the sounds produced by meteors. Ph.D. Dissertation, Ann Arbor, University of Michigan, MI (1974)

D.O. ReVelle, On meteor-generated infrasound. J. Geophys. Res. 81, 1217-1230 (1976)

D.O. ReVelle, in Annals of the New York Academy of Sciences, Near-Earth Objects-The United Nations International Conference, ed. by J.L. Remo. Historical detection of atmospheric impacts by large bolides using acoustic-gravity waves, vol 822 (New York Academy of Sciences, New York, NY, USA, 1997), pp. 284-302

D.O. ReVelle, R.W. Whitaker, Infrasonic detection of a Leonid bolide: 1998 November 17. Meteorit. Planet. Sci. 34, 995-1005 (1999)

R. Swinbank, A.A. O’Neill, Stratosphere-Troposphere Data Assimilation System. Mon. Weather Rev 122, 686-702 (1994)

R.J. Weryk, P.G. Brown, W.N. Edwards, Z. Krzeminski, S.H. Nudds, A. Domokos, D.L. Welch, The Southern Ontario meteor camera network. Earth Moon Planets (2007) this issue 